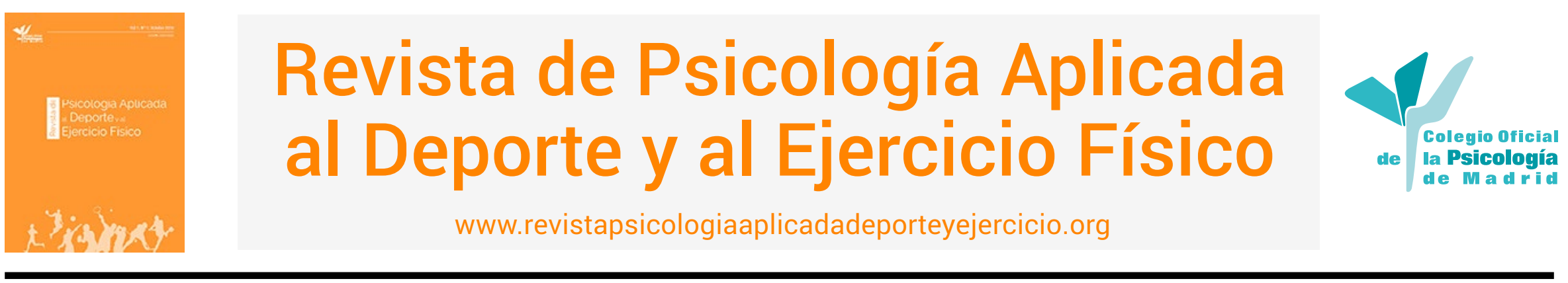

\title{
Barreras psicológicas en el rendimiento del remoergómetro
}

\author{
Ignasi Navarro Soria' ${ }^{1}$ Ana Sempere Sempere' ${ }^{1}$ y Borja Costa López ${ }^{2}$ \\ 'Universidad de Alicante, España \\ 2Universidad Miguel Hernández, España
}

RESUMEN: En la alta competición, es de vital importancia el análisis de los factores que puede afectar al rendimiento. En el deporte de remo, el remo indoor es un aparato utilizado para valorar el nivel de rendimiento deportivo de los remeros. Las pruebas de valoración del remoergómetro, suponen situaciones estresantes para los deportistas, produciendo reacciones negativas que impiden que el remero/a refleje completamente todo su potencial físico. Por ello, esta investigación tiene como objetivo analizar las barreras/resistencias que influyen en el rendimiento del remero/a y la percepción de utilidad de la prueba de valoración para los deportistas, así como el grado de efectividad de un programa de intervención psicológica sobre la ansiedad estado, como estrategia principal para mejorar el rendimiento deportivo. Cuatro equipos de remo fueron escogidos para la investigación, un total de 27 participantes de la categoría juvenil y absoluta, 19 mujeres y 8 hombres de entre 16-44 años. Se realizaron dos evaluaciones (pre-intervención y post-intervención), suministrándole a los deportistas unos cuestionarios antes de la prueba de valoración en el remoergómetro: el CPRD, el STAl y el SDQ-II. Durante la intervención psicológica se aplicó la técnica de relajación muscular progresiva de Jacobson y el fomento de las autoinstrucciones. Los resultados obtenidos mostraron la efectividad de las estrategias de afrontamiento aplicadas durante un periodo de dos meses, alcanzando unos resultados satisfactorios en la prueba de valoración en el remoergómetro, con unos niveles de ansiedad estado inferiores y un aumento en los niveles de activación, apariencia física, importancia en la prueba y rendimiento. Se concluye que la ansiedad estado es una variable influyente en el rendimiento deportivo y que, mediante el entrenamiento psicológico, se puede reducir, mejora del rendimiento deportivo y otras variables relacionadas.

PALABRAS CLAVES: ansiedad estado, autoconcepto, remo indoor, estrategias de afrontamiento, rendimiento.

\section{Psychological barriers in rowing ergometer performance}

ABSTRACT: In high-level competition, analysis of factors that can affect performance is of vital importance. In rowing, indoor rowing is used to assess the level of sport performance of rowers. The assessment tests of the rowing ergometer represent stressful situations for athletes which cause negative reactions that prevent rowers from fully developing their full physical potential. This research was therefore aimed at analyzing the barriers/resistances that influence the performance of rowers and the perception they have of the value of the assessment test, as well as the degree of effectiveness of a psychological intervention program on state anxiety as the main strategy to improve sports performance. Four rowing teams, a total of 27 participants from the youth and absolute category, 19 women and 8 men aged 16-44 years, were selected for the research. Evaluations were performed before and after intervention, and athletes completed before the rowing ergometer assessment test the CPRD, STAI, and SDQ-II questionnaires. During psychological intervention, the Jacobson's progressive muscle relaxation technique was applied, and self-instructions were promoted. The results showed the effectiveness of the coping strategies applied for two months, as satisfactory results were achieved in the rowing ergometer assessment test with decreased state anxiety levels (from $M=37.15$ to $M=21.67 ; p=.01$ ) and increased activation levels, physical appearance, importance in the test, and performance. It is concluded that state anxiety is an influential variable in sports performance and that it may be reduced by psychological intervention, thus improving sports performance and other related variables.

KEYWORDS: state anxiety, self-concept, indoor rowing, coping strategies, performance.

\footnotetext{
Ignasi Navarro Soria (iD https://orcid.org/0000-0001-5966-9604 es Psicólogo en la Universidad de Alicante.

Ana Sempere Sempere (iD https://orcid.org/0000-0002-5494-298X es Entrenadora Deportiva del Club Náutico Santa Pola.

Borja Costa López (iD https://orcid.org/0000-0002-6658-768X es Psicólogo en la Universidad Miguel Hernández.

La correspondencia sobre este artículo debe enviar a Ignasi Navarro Soria. Universidad de Alicante. San Vicente del Raspeig / Sant Vicent del Raspeig, Alicante. E-mail:
}

(cc) EY-No-ND Este es un artículo Open Access bajo la licencia 


\section{Barreiras psicológicas no desempenho do remoergómetro}

RESUMO: Em alta competição, uma análise de cada um dos fatores que podem afetar o desempenho é de vital importância. No remo, o remo indoor é um aparelho usado para avaliar o nível de desempenho desportivo dos remadores. Os testes de avaliação do remoergómetro implicam situações de tensão para os atletas, produzindo reações negativas que impedem o/a remador/a de refletir completamente todo o seu potencial físico. Assim, esta investigação tem como objetivo analisar as barreiras/resistências que influenciam o desempenho do/a remador/a e a perceção da utilidade do teste de avaliação para os atletas, bem como o grau de eficácia de um programa de intervenção psicológica na ansiedade-estado como a principal estratégia para melhorar o desempenho desportivo. Quatro equipas de remo foram escolhidas para a investigação, num total de 27 participantes da categoria juvenil e absoluta, 19 mulheres e 8 homens com idades entre os 16 e 44 anos. Foram realizadas duas avaliações (pré-intervenção e pós-intervenção), tendo sido fornecidos questionários aos atletas antes da prova de avaliação no remoergómetro: o CPRD, o STAI e o SDQ-II. Durante a intervenção psicológica, foi aplicada a técnica de relaxamento muscular progressivo de Jacobson e a promoção de autoinstruções. Os resultados obtidos demonstraram a eficácia das estratégias de adaptação aplicadas, durante um período de dois meses, alcançando resultados satisfatórios na prova de avaliação no remoergómetro, com menores níveis de ansiedade-estado e um aumento nos níveis de ativação, aparência física, importância na prova e desempenho. Conclui-se que a ansiedade-estado é uma variável que influencia o desempenho desportivo e que, através do treino psicológico, pode ser reduzida, melhorando o desempenho desportivo e outras variáveis relacionadas.

PALAVRAS-CHAVE: ansiedade-estado, autoconceito, remo indoor, estratégias de adaptação, desempenho.

Artículo recibido: 13/04/2020 | Artículo aceptado: 02/06/2020

El remo, es un deporte muy exigente a nivel físico y psicológico (Hagerman et al., 1978). Entre las diferentes modalidades del remo se encuentra el remoergómetro, una máquina que simula la acción de remar y que se utiliza principalmente para la preparación física del deportista. El remo indoor es una herramienta esencial para evaluar en qué estado físico se encuentra el remero/a, a través de éste se realizan las pruebas de valoración, indicándonos el tiempo de la realización de la prueba, los watts generados, el parcial realizado, el ritmo de paladas, entre otros parámetros. Los resultados de esta prueba indican, principalmente, el nivel de rendimiento del remero/a (Pierna et al., 2008).

Diferentes investigaciones estudian las variables más físicas del rendimiento en el remo en general y en el remoergómetro en concreto (Schabort et al., 1999). Se ha estudiado patrones motores (Elliott et al., 2002), características físicas de los remeros (Akça, 2014; Huang et al., 2007; Silva 2019), la influencia de la capacidad cardiorrespiratoria (Astorino et al., 2012), la fuerza en las extremidades implicadas en la remada (Gentil et al., 2017; Perna et al., 2017), la edad del deportista (Baker y Tang, 2010; Galloway et al., 2002) y la cinemática (Zainuddin et al., 2019), entre otras cuestiones que afectan al rendimiento del deportista. En cambio, no encontramos literatura científica que verse en torno a los aspectos psicológicos, que, en concreto en el remoergómetro, pueden influir sensiblemente en la mejora de diferentes aspectos emocionales a la hora de afrontar la práctica deportiva y en consecuencia, su rendimiento.

Si tenemos en cuenta que esta herramienta de entrenamiento, en muchos equipo de alto rendimiento, son la medida para la selección de los remeros más preparados para componer el equipo de cara a una competición, a la mayoría de los remeros la prueba le supone una situación estresante (Filaire et al., 2001; Jones y Hardy, 1990; Márquez, 2004) que pueden provocar alteraciones del funcionamiento psicológico (menor capacidad de concentración, pérdida del foco atencional, aumento de la ansiedad estado) y fisiológico (incremento de la tasa cardiaca, incremento de la tensión muscular, etc.). Como consecuencia, el remero/a no desarrolla todo su potencial físico, reflejando un rendimiento menor a sus posibilidades (García et al., 2008; Kendall et al., 2011).

Diferentes variables han sido estudiadas en relación con el rendimiento de los deportistas, concretamente en el caso del atletismo, han sido destacadas la ansiedad cognitiva, la ansiedad somática y la autoconfianza como tríada de factores relacionados con las emociones en pre-competición (Jaenes y Caracuel, 2005; Sánchez et al., 2012). Investigaciones recientes (García-Más et al. 2011; Marco et al., 2007; Ries et al., 2012; Scanlan et al., 2005), afirman que dentro de las variables psicológicas influyentes en la productividad deportiva para la consecución del éxito destacan la motivación, la atención, el estrés, la ansiedad, la autoconfianza, los estados de ánimos, el autocontrol y la autorregulación, la cohesión, las habilidades interpersonales o el ajuste emocional. De igual manera, un número importante de investigaciones (Anshel, 1995; Auweele et al., 1993; Gutiérrez et al., 1997; Ortín et al., 2013; Pozo, 2007; Roberts, 2001; Ruiz-Juan et al., 2015; Smith, 1989) buscan relaciones entre ansiedad y rendimiento deportivo, destacando algunas de ellas entre sus resultados, que el factor ansiedad precompetitiva, puede condicionar el rendimiento en el deporte. 
En las investigaciones anteriores se muestra la ansiedad como factor determinante del rendimiento deportivo. Saz (2000) y Campos et. al. (2017) describen la ansiedad como un estado emocional de tensión nerviosa y de miedo intenso, caracterizada por síntomas somáticos como temblor, inquietud, sudoración hiperventilación, palpitaciones, etc., cuyos síntomas cognitivos son inquietud psíquica, hipervigilancia, pérdida de concentración y distorsiones cognitivas. De igual modo, Sierra et al. (2003) describen la ansiedad como un estado de agitación e inquietud desagradable caracterizado por la anticipación del peligro, el predominio de síntomas psíquicos y las sensaciones de catástrofe o de peligro inminente. Es decir, es una combinación entre síntomas cognitivos y fisiológicos, en la que se manifiesta una reacción de sobresalto, donde el individuo trata de buscar una solución al peligro. En esta misma línea, Schlatter (2003) y Aldás et al. (2018) refieren a la ansiedad como una sensación subjetiva que presentamos ante situaciones que valoramos como amenazantes o riesgo, ya sea por carácter novedoso o imprevisto o por la intensidad del estímulo. Investigadores más recientes (Aldás et al., 2018; Torrez y Núñez, 2012) la definen como un fenómeno negativo que incluye sensaciones de nerviosismo, preocupación y aprensión, las mismas que están relacionadas con la activación o desactivación del organismo sobre todo en situaciones de alerta.

Algunas investigaciones han constatado una relación negativa entre la ansiedad y el rendimiento. Así, según apuntaba Spielberger (1966), los deportistas con un alto grado de ansiedad rasgo tendrán, a su vez, un mayor nivel de ansiedad estado y, en consecuencia, mayor riesgo de rendir por debajo de su potencial en la competición. Más tarde, Wang, Marchant, Morris y Gibbs (2004) coincidieron con otras investigaciones realizadas con deportes de equipo en las que, la ansiedad estado precompetitiva influyó negativamente en el rendimiento de los deportistas. Investigaciones posteriores (Cano et al., 2009) reafirmaron la investigación anterior, constatando que la ansiedad estado precompetitiva afectó negativamente al rendimiento de los jugadores de baloncesto, aumentando el número de balones perdidos y disminuyendo la eficacia de los tiros.

Para paliar los efectos negativos de la ansiedad precompetitiva sobre el rendimiento, existen varias técnicas que los deportistas administran en sus competiciones, para que el rendimiento sea óptimo y su capacidad física, técnica y cognitiva no se altere en momentos de ansiedad, adversidad o durante la ejecución de una determinada acción, estas técnicas son conocidas como estrategias de afrontamiento (Portilla del Hierro, 2016; Weinberg, 2007). Así, el afrontamiento representa aquellos esfuerzos cognitivos y conductuales constantemente cambiantes que se desarrollan para mane- jar las demandas específicas externas y/o internas que son evaluadas como excedentes o desbordantes de los recursos del individuo (Carrasco et al., 2010; Lazarus y Folkman, 1984).

Urra (2014) concluyó con los resultados de su investigación, que el entrenamiento de estrategias de afrontamiento constituye una intervención útil para reducir los niveles de ansiedad precompetitiva. Así lo confirma una investigación reciente (Olmedilla et al., 2018) que corroboró, que las jugadoras de fútbol que recibieron entrenamiento psicológico mejoraron sus recursos y habilidades psicológicas frente a aquellas que no lo realizaron. El empleo de algunas estrategias de afrontamiento los días previos a la competición, tales como autoinstrucciones para mejorar el autoconcepto, visualización, o estrategias de afrontamiento del estrés, focalizando la atención en los aspectos que se puede controlar, se han mostrado muy útiles en estudios con deportistas de diferentes niveles y modalidades deportivas (Bertollo et al., 2009; Carretero, Moreno-Murcia, González, Ponce y Gimeno, 2012; Gould et at., 1993; Hatzigeorgiadis et al., 2009; Mamassis y Dogamis, 2004). En una investigación realizada con jóvenes tenistas de alta competición, Carrasco et al. (2010), concluyeron que la calma emocional (por medio de la relajación, visualización, respiración, entre otras técnicas) fue una estrategia de afrontamiento eficaz. Asimismo, Urra (2014) concluyó que las técnicas de respiración diafragmática y autodiálogo, actúan de forma inmediata sobre aquellos indicadores que los deportistas asociaron mayormente con el estado de ansiedad.

Debido a la importancia que tiene la ansiedad precompetitiva sobre el rendimiento deportivo y las consecuencias negativas que pueden acarrear en el remero/a para la obtención de un mejor resultado en la prueba de evaluación en el remo indoor, se persigue analizar resistencias/barreras que influyen en el rendimiento del remero/a y la percepción de utilidad que tiene la prueba para los remeros, así como aplicar y analizar el grado de efectividad de un programa de intervención psicológica, centrada en la técnica de relajación (Duval, 2010; Smith, 1992) y autoinstrucciones (Bertollo et al., 2009; Carretero et al., 2012; Hatzigeorgiadis et al., 2009), sobre variables psicológica como la ansiedad estado, la motivación, la activación, la concentración o percepción que los deportistas tienen sobre si mismos ante la prueba precompetitiva. De hecho, partimos de la hipótesis de que, si se da una mejora significativa de los niveles de ansiedad, con los que el remero afronta la prueba precompetitiva del remoergómetro, se conseguirá reducir alguna de las barreras que reducen la autoevalaución de su pontencial para superarse, obteniendo de esta manera una mejora en el rendimiento. 


\section{Método}

\section{Diseño}

Diseño experimental de pre y post intervención en un solo grupo aplicado de forma individual.

\section{Participantes}

La muestra incluye 27 remeros con una edad comprendida entre los 16 y 44 años $(M=21.30, D . T .= \pm 8.36)$ de nivel federativo de la modalidad llaut de banco fijo del mediterráneo, siendo 19 mujeres (70.37\%) y 8 hombres (29.62\%). Se seleccionó a los equipos juveniles y absoluto, tanto masculino como femenino, por la igualdad de la distancia en la prueba de evaluación en el remoergómetro (2000m). Todos los deportistas fueron informados de los objetivos del estudio y proporcionaron su consentimiento para participar en este estudio. Del total de la muestra, 11 han sido remeras de la categoría juvenil femenino (40.74\%), 4 remeros juvenil masculino (14.81\%), 8 remeras absoluto femenino (29.62\%) y 4 remeros absoluto masculino (14.81\%). Los participantes entrenan 6 días a la semana, de lunes a sábado, dos horas diarias. Este entrenamiento es el preparatorio para competiciones autonómicas y nacionales.

La puesta en práctica y proporción de refuerzos positivos, se llevó a cabo entre un psicólogo doctor en Psicología, con formación clínica y conocimientos como psicólogo general sanitario colegiado y 20 años de ejercicio profesional, con una dilatada experiencia en diferentes ámbitos educativos, en coordinación con una entrenadora deportiva graduada en Ciencias de la Actividad Física y Deporte y especialista, titulada en Master en alto rendimiento deportivo, con experiencia en competición como remera federada del Club Náutico de Santa Pola y 11 años de bagaje como entrenadora deportiva en diferentes categorías.

\section{Instrumentos}

A continuación, se describen los diferentes instrumentos de medición que se han implementado para la recogida de datos, según la variable psicológica que miden:

\section{Características psicológicas del deportista que influyen en el rendimiento del remero}

Se aplicó el cuestionario Características Psicológicas relacionadas con el Rendimiento Deportivo (CPRD) (Gimeno et al., 2001). Este cuestionario consta de 55 ítems evaluando cuatro dimensiones: concentración (8), nivel de activación (26), motivación (6) y percepción de utilidad (10), con una escala de respuesta tipo Likert cuyo rango de respuesta va desde 1 (totalmente de acuerdo) a 5 (totalmente en desacuerdo). Así mismo, se valoraron dos ítems relacionados con el esfuerzo al realizar la prueba e importancia de la prueba, con una escala de respuesta tipo Likert cuyo rango de respuesta va desde 1 (máximo/a) a 5 (mínimo/a). El cuestionario tiene una buena consistencia interna en su conjunto obteniendo una puntuación de .85 en el coeficiente Alfa de Cronbach (Gimeno et al., 2001)

\section{Ansiedad estado y rasgo}

La ansiedad estado y rasgo se midió utilizando la versión española del Cuestionario de Ansiedad Estado-Rasgo (State-Trait Anxiety Inventory, STAl; Spielberger et al., 1970). Un total de 40 ítems conforman el autoinforme STAI para evaluar dos factores: ansiedad estado (AE) y ansiedad rasgo (AR). Cada concepto conforma un total de 20 ítems utilizando un sistema de respuesta tipo Likert, en la que $0=$ nada/ casi nunca; 1 = algo/a veces; $2=$ bastante/ a menudo; $3=$ mucho/casi siempre. El cuestionario tiene una buena consistencia interna en sus dos subescalas (ansiedad estado y rasgo), que oscila entre .84 y .93, respectivamente, en la adaptación española (Fonseca-Pedrero et al., 2012).

\section{Autoconcepto}

El autoconcepto se evaluó a través de una versión reducida del cuestionario Self-Description Questioannaire II (SDQII) basado en el modelo multidimensional propuesto por Shavelson et al. (1976). Está compuesto por 33 ítems que hacían referencia a 7 dimensiones del autoconcepto: a) seis no académicas (habilidad física (4 items), apariencia física (4 items), relaciones con el mismo sexo (5 items), relaciones con el sexo opuesto ( 4 items), sinceridad-veracidad (5 items) y estabilidad emocional (5 items)), que evalúan, respectivamente, las habilidades en el ámbito físico o deportivo, el atractivo físico, las relaciones percibidas respecto a diferentes agentes sociales, la sinceridad y el bienestar emocional; b) una que evalúa la satisfacción del sujeto consigo mismo (self) (6 items). Sigue un formato de respuesta tipo Likert con un rango de 1 (falso) a 6 (verdadero). El cuestionario presenta una consistencia interna que oscila entre .70 y .84, en la adaptación española (García-Fernández et al, 2016). 


\section{Prueba de valoración}

La prueba de valoración se registró en una máquina remo indoor Modelo D con el monitor de rendimiento PM5, ofreciendo datos comparables y precisos de cada palada realizada durante la prueba de $2000 \mathrm{~m}$. Las variables medidas de las que se obtiene una puntuación global de rendimiento son; las paladas por minuto (ritmo), la potencia (watts), la velocidad en cada parcia de 500 metros y el tiempo empleado en realizar la totalidad de la prueba (Pierna et al., 2008).

\section{Procedimiento}

Este trabajo es fruto de la colaboración entre el Departamento de Psicología Evolutiva y Didáctica de la Universidad de Alicante y el Club Náutico de Santa Pola, en el marco de la realización de un proceso de intervención individual. Posteriormente, para la selección de la muestra, el club escogió a remeros de las categorías juvenil y absoluto (masculino y femenino) de forma aleatoria por accesibilidad, y junto con el psicólogo de referencia, se informó de las características de la investigación, incluyendo la obtención de un consentimiento para su participación. El periodo de la temporada en el que se encuadra la investigación es el pre-competitivo, periodo en el que los entrenadores deportivos de llaut, llevan a cabo la selección de los deportistas que formaran parte del equipo de Llaut Mediterráneo que representan al Club Santa Pola en el campeonato de España. De hecho, la selección para dicha competición se lleva a cabo en base a los resultados obtenidos en el remoergómetro y que supone el posttest de esta investigación. Toda la información recogida es tratada de forma anónima y confidencial, respetando la Ley Orgánica 3/2018, 5 de diciembre, de Protección de Datos y Garantía de los Derechos Digitales (Ley Orgánica 3/2018, 2018). Tras acordar las fechas de la intervención, los profesionales (psicólogo y entrenadora) llevaron a cabo la intervención en las instalaciones del club acondicionadas para tales entrenamientos.

La intervención consta de 16 sesiones aplicadas de forma individual a cada deportista con una periodicidad de dos veces por semana durante 8 semanas y una duración de $30 \mathrm{mi}-$ nutos por sesión. Estas fueron llevadas a cabo siempre por la misma entrenadora en coordinación con el mismo psicólogo.

En esta intervención se realizaron dos evaluaciones, una al inicio (pre-intervención) y otra al final (post-intervención), en las cuales se analizaron las resistencias que influyen en la obtención de un mejor resultado, la ansiedad rasgo y esta- do, y el autoconcepto. El cuestionario CPRD de rendimiento deportivo se cumplimentó el día antes de la prueba de valoración en el remoergómetro, el resto de los cuestionarios (SDQ-II y STAI) se realizaron minutos previos a la misma, respectivamente. La estrategia se implementó previo al horario de entrenamiento ordinario, las 18 horas, en el gimnasio del Club Náutico, espacio reservado en exclusiva para los participantes del estudio.

La estructura de cada sesión de la intervención consistió en que antes del entrenamiento físico se realizaba la relajación progresiva de Jacobson (González y Andrés, 2019), trabajando tensión-relajación muscular de las partes más utilizadas por el remero/a (miembros superiores, zona cervical, abdomen y miembros inferiores) en una sala aislada y con música ambiental, está parte de la sesión se implementa para todos los deportistas por igual. Durante el entrenamiento se trasladaron reforzadores positivos diseñados y previstos en un repositorio que se pone a disposición de la entrenadora (ej. Has colocado muy bien la pala en el ataque, has buscado el agua y has entrado en el momento preciso, lo que ha llevado a un ataque directo o has hecho una subida de velocidad muy buena, tu actitud ha sido excelente, has conseguido que el equipo no caiga en los momentos más duros) y tras el entrenamiento físico se instruía en autoinstrucciones (ej. Debes mantener la con concentración en los momentos de mayor tensión, esto lo trasmites al equipo y lo fortalece o la intensidad con la que trabajo es clave para el éxito del equipo) (García-Naveira, 2017; Labrador, 2014), a cada remero de forma aislada, teniendo en cuenta sus características como deportista.

\section{Análisis de los datos}

Se utilizó el programa IBM SPSS Statistics v25 para el procesamiento estadístico de los datos. Se calculó la media (M) y desviación típica (DT) como medidas de tendencia central para el análisis descriptivo de las variables mencionadas anteriormente. Por otro lado, se llevó a cabo un análisis de diferencias pre-post a través de la aplicación de la prueba T-student para muestras relacionadas, utilizando un nivel de significación a =.05.

\section{Resultados}

En la Tabla 1, se presentan las medias de las puntuaciones obtenidas en la pre-evaluación y en la post-evaluación 
Tabla 1. Análisis de diferencias pre y post intervención con remeros a través del estadístico de contraste t para muestras relacionadas $(n=27)$

\begin{tabular}{|c|c|c|c|c|c|c|}
\hline & \multicolumn{2}{|c|}{ Pre } & \multicolumn{2}{|c|}{ Post } & \multirow{2}{*}{$t$} & \multirow{2}{*}{$p$} \\
\hline & M & $D T$ & M & $D T$ & & \\
\hline Nivel de concentración & 21.59 & 6.08 & 20.59 & 6.54 & 1.11 & .28 \\
\hline Nivel de activación & 67.63 & 6.86 & 71.59 & 7.67 & -2.85 & $.01^{*}$ \\
\hline Nivel de motivación & 15.74 & 4.78 & 16.22 & 4.75 & -.70 & .49 \\
\hline Nivel de utilidad & 24.63 & 4.31 & 24.89 & 4.34 & -.31 & .76 \\
\hline Apariencia física & 16.15 & 4.08 & 17.44 & 3.30 & -2.14 & $.04^{*}$ \\
\hline Self & 26.52 & 5.68 & 27.93 & 5.20 & -2.31 & $.03^{*}$ \\
\hline Sinceridad-veracidad & 25.59 & 2.79 & 24.52 & 3.68 & 2.12 & $.04^{\star}$ \\
\hline Habilidad física & 17.70 & 3.56 & 17.93 & 3.35 & -.70 & .49 \\
\hline Estabilidad emocional & 20.41 & 5.80 & 19.85 & 5.00 & .79 & .44 \\
\hline Relaciones sexo opuesto & 18.74 & 3.81 & 19.22 & 3.14 & -.97 & .34 \\
\hline Relaciones mismo sexo & 24.37 & 4.46 & 24.56 & 4.49 & -.43 & .67 \\
\hline Ansiedad rasgo & 23.70 & 8.23 & 22.11 & 8.14 & 1.50 & .15 \\
\hline Ansiedad estado & 37.15 & 13.42 & 21.67 & 13.56 & 5.76 & $.01 *$ \\
\hline Esfuerzo al realizar la prueba & 1.33 & .48 & 1.22 & 0.51 & 1.00 & .33 \\
\hline Importancia de la prueba & 1.00 & .00 & 1.19 & 0.48 & -1.99 & .06 \\
\hline Resultado de la prueba (rendimiento) & 492.63 & 44.82 & 496.63 & 46.58 & 2.31 & $.03^{\star}$ \\
\hline
\end{tabular}

* Nivel de significación $=.05$

en cada una de las variables. Al analizar las puntuaciones obtenidas, tal y como se muestra en la Tabla 1, se puede observar que existen diferencias estadísticamente significativas en 6 variables: activación $(t(26)=-2.85 ; p=.01)$, ansiedad estado $(t(26)=5.76 ; p=.01)$, percepción de apariencia física $(t(26)=-2.14 ; p=.04)$, self $(t(26)=-2.31 ; p=.03)$, sinceridad-veracidad $(t(26)=2.12 ; p=.04)$, resultado de la prueba (rendimiento) $(t(26)=2.31 ; p=.03)$.

\section{Discusión}

El propósito de esta investigación ha sido comprobar el grado de efectividad de un programa de intervención psicológica, centrado en la técnica de Relajación Progresiva de Jacobson (Smith, 1992) y las autoinstrucciones positivas (Bertollo et al., 2009; Carretero et al., 2012; Gould et at., 1993; Hatzigeorgiadis et al., 2009; Mamassis y Dogamis, 2004), con la finalidad de reducir la ansiedad como una de las barreras psicológicas que impiden un mejor rendimiento del deportista.

En base a los resultados obtenidos, se puede afirmar que se cumple la hipótesis inicial, ya que de los datos se des- prende una mejora significativa en diferentes medidas psicológicas, sobretodo en ansiedad estado, medida esta previo a la realización de la prueba. De ello entendemos, que la mejora que se observa en el rendimiento ante el remoergómetro, puede ser resultado de la mejora en la activación y la reducción de la ansiedad estado con la que afrontan la prueba el grupo de remeros que componen la muestra.

Tal y como sucediera en estudios previos, con deportistas de otras disciplinas, como son las artes marciales (Salas y Salazar, 2004) o el futbol (Mosconi, Correche, Rivarola y Penna, 2007), el empleo de técnicas de relajación logra un efecto positivo en el rendimiento, debido a que esta estrategia contribuye a la disminución de los niveles de ansiedad y favorece, un estado psicológico positivo, de cara a afrontar situaciones competitivas. De hecho, otros muchos autores (Ángeles y Hernández, 2007; Latinjak et al., 2009; Zinsser et al., 2006) concluyen en sus investigaciones que las técnicas de relajación en combinación con destrezas en autorregulación psicológica a través de autoinstrucciones (la visualización, la autosugestión, la activación, control de la respuesta de ansiedad, autoconversación), favorecen significativamente la efectividad en el desarrollo de habilidades estratégicas y por tanto, la reducción de la ansiedad en el 
deportista y mejora de su rendimiento. Por lo que nuestros datos, vendrían a apoyar los resultados de estas investigaciones previas.

Las medidas psicológicas que mejoran tras el programa de intervención son la activación, la imagen y las creencias de capacidad que los remeros tienen sobre sí mismos, ligado a un descenso importante de la ansiedad estado. Todo ello entendemos que, está unido a unas expectativas positivas sobre la capacidad en la ejecución de la prueba y en su conjunto, a la confianza que tienen en sí mismos para mejorar el rendimiento en la prueba (Martens, 1987; Sánchez-Hernández y López-Fernández, 2005; Vargas, 2015).

Si hacemos un análisis más pormenorizado, se puede afirmar que teniendo en cuenta que la ansiedad está estrechamente relacionada con el arousal, la conducta final tendrá una dimensión que podrá ser dependiente del nivel de activación (Navarro-Guzman, Amar, y González-Ferreras, 1995), lo que justifica que este nivel de activación mejore y por tanto, favorezca el funcionamiento del deportista ayudándole a rendir al máximo dentro de sus posibilidades (Buceta, 1994).

Resulta interesante destacar que a pesar de poder observarse un aumento tanto en la variable Importancia del test como Apariencia física, medidas ligadas a la trascendencia que ofrece el deportista a la actividad que realiza, no se han observado diferencias estadísticamente significativas en la variable Nivel de motivación pre-test y post-test, siendo otra de las grandes variables psicológicas que tienen una notable importancia en el deporte de competición (Auweele et al., 1993; Buceta, 1990 y 1996; García et al., 2006; Gil, Capafons y Labrador, 1993; Gimeno et al., 2007; Loehr, 1984; Mahoney et al., 1987; Roberts, 2001).

Finalmente, otro aspecto a considerar es la variable Sinceridad-veracidad, en la que se observa una diferencia estadísticamente significativa entre el pre-test y post-test, resultando menor en el post-test. Este hecho implica que los remeros se muestran menos sinceros en sus respuestas al cuestionario que mide la ansiedad estado, conforme se sienten más observados y crece su deseabilidad social, pudiendo así alterar alguna de sus respuestas para satisfacer las expectativas de la entrenadora.

Para finalizar, el que los remeros hayan mejorado el estado emocional antes de la prueba, enfrentándose a ésta con mayor autoconfianza, autocontrol y seguridad, ha supuesto que estos logren unos resultados más satisfactorios a nivel deportivo. Estos resultados son coincidentes con la mayoría de la literatura científica, que indica que niveles altos de ansiedad competitiva influyen de manera negativa tanto en el rendimiento como en el disfrute y en el bienestar físico (García-Más et al. 2011; Ortín et al., 2013; Ries et al., 2012; Scanlan et al., 2005).

\section{Conclusión}

Teniendo en cuenta los datos obtenidos, se puede afirmar que las estrategias de afrontamiento basadas en el método de Relajación Progresiva de Jacobson y las autoinstrucciones positivas, han resultado efectivas para reducir los niveles de ansiedad precompetitiva en remeros antes de una prueba de evaluación en el remo indoor. Además, su implementación ha supuesto beneficios en la mejora de la autoconfianza de los remeros, aumentando las puntuaciones en su percepción física. Así mismo, tras la intervención psicológica, se han reflejado mayores niveles de activación, pudiendo considerarse este estado de activación como "positivo", debido a su estrecha relación con la ansiedad y teniendo en cuenta que ésta última, ha sido óptima ante la prueba de evaluación en base a los resultados alcanzados.

\section{Aplicaciones prácticas}

A raíz de estos resultados, se destaca la importancia de incorporar un trabajo psicológico en el programa anual de entrenamiento de un equipo deportivo, para reducir los efectos de la ansiedad precompetitiva ante cualquier competición o prueba de evaluación del rendimiento deportivo. Así como para incidir en otras variables influyentes en el rendimiento deportivo como son el nivel de activación y la autoconfianza, entre otras.

La preparación psicológica de los deportistas debe integrarse en el conjunto de su preparación global, como un elemento más que tiene que interactuar, apropiadamente, con las parcelas física, técnica y táctico/estratégica. Considerando todas las vías de obtención de información e influencia psicológica que, permitan comprender y mejorar su funcionamiento deportivo (Buceta, 1998).

Así mismo, resulta imprescindible aplicar refuerzos positivos que los deportistas conviertan en autoinstrucciones, para que se sientan competentes con las tareas que realizan, y en consecuencia, seguros y confiados para poder afrontar con éxito las competiciones o pruebas de evaluación que se les plantee. En caso de remeros, sería importante resaltar tanto la progresión conseguida a nivel técnico, destacando aquellos gestos que impliquen una mejora en el rendimiento deportivo, por ejemplo: el uso de las piernas, el ataque rápido o la colocación del cuerpo durante la recuperación, como las consecuencias que su mejora suponen para el rendimiento grupal, en modalidades que impliquen dos personas o más, destacando aspectos relacionados con el ritmo de paladas o la intensidad en el agua.

Los datos expuestos en este estudio pueden aportar gran valor al trabajo profesional de los técnicos deportivos de 
remo ya que ayudaría a detectar la ansiedad precompetitiva de los deportistas de manera precoz. Esto puede ser clave para anticiparse a la situación y trabajar con los remeros aspectos de refuerzo personal y deportivo que eviten posibles rechazos hacia la práctica de remo indoor. Además, la puesta en práctica de los métodos expuestos en el artículo, por parte del técnico, pueden ayudar a maximizar las capacidades deportivas de los deportistas cuando se enfrente a pruebas deportivas concluyentes, como podría ser la realización de una prueba de evaluación para formar parte del equipo para el Campeonato Nacional.

\section{Posibles limitaciones}

La investigación realizada presenta algunas limitaciones desde el punto de vista metodológico y de la muestra, entre las que destacan las características de los participantes de la muestra, que sí bien habría sido más apropiado seleccionar únicamente a una categoría, reduciendo de esta forma el rango de edad de la muestra (16-44) siendo está más homogénea, se ha tenido que escoger dos categorías (absoluto y juvenil) para aumentar la muestra de la investigación. Eso sí, ambas con la misma distancia en metros a recorrer en la prueba de evaluación.

En segundo lugar, no ha sido posible la comparación entre sexos debido a la diferencia de participación entre hombres y mujeres, siendo estas últimas el doble.

Finalmente, a nivel metodológico, cabe destacar una posible interferencia "negativa" en los resultados, debido a que la variable "sinceridad-veracidad" de los sujetos resultó menor en el post-test.

\section{Perspectivas futuras}

En base a lo expuesto, futuras investigaciones podrían analizar si realmente existen diferencias entre sexos en el nivel de ansiedad precompetitiva en la prueba de evaluación en el remo indoor, utilizando una muestra más homogénea para reducir la diferencia de edad entre los sujetos, observando así mismo, si la edad es un factor condicionante del nivel de ansiedad precompetitiva.

También debiera estudiarse si toda la mejora se debe exclusivamente a la óptima gestión de la ansiedad precompetitiva o si el propio entrenamiento ha supuesto una mejora sustancial en el rendimiento de los deportistas. Para ello deriva replicarse la investigación incluyendo un grupo control que, llevase a cabo el mismo entrenamiento deportivo, sin el trabajo psicológico.
Además, sería interesante analizar de forma más exhaustiva otras variables influyentes en el rendimiento deportivo, tales como la motivación o el estado de ánimo, observando el grado de influencia de ambas variables y actuando sobre éstas.

Por último, sería interesante y, tal vez más adecuado, emplear una prueba de ansiedad específica para actividades deportivas como podría ser el Test de Ansiedad Precompetitiva Versión Revisada (CSAI-2R) de Cox et al., (2003).

\section{Referencias}

Aldás, A. C., Saltos, L. y Robayo, J. (2018). Influencia de la ansiedad en el rendimiento deportivo en futbolistas. Revista de Ciencia y Tecnologia, 18(19), 210-202

Ángeles, R. A. y Hernández, R. (2007). Técnicas de relajación de Jacobson como herramienta en la psicología deportiva para favorecer el rendimiento deportivo en el equipo de fútbol categoría 88 del centro de formación Pachuca [Tesis Doctoral no publicada]. Universidad Autónoma del Estado de Hidalgo, México.

Akça, F. (2014). Prediction of Rowing Ergometer Performance from Functional Anaerobic Power, Strength and Anthropometric Components. Journal of Human Kinetics, 41(1), 133-142. https://doi.org/10.2478/hukin-2014-0041

Anshel, M. (1995). Anxiety. En T. Morris y J. Summers (Eds.), Sport Psychology: Theory, Applications \& Issues (pp. 29-62). John Wiley \& Sons.

Astorino, T. A., Allen, R. P., Roberson, D. W. y Jurancich, M. (2012). Effect of high-intensity interval training on cardiovascular function, V02max, and muscular force. The Journal of Strength \& Conditioning Research, 26(1), 138- 145.

Auweele, Y. V., De-Cuyper, B., Van-Mele, V. y Rzewnicky, R. (1993). Elite Performance and Personality: From Description and Prediction to Diagnosis and Intervention. En R. N. Singer, M. Murphey y L. K. Tennant (Eds.), Handbook of Research on Sport Psychology (pp. 257-299). Macmillan.

Baker, A. B. y Tang, Y. Q. (2010). Aging performance for masters records in athletics, swimming, rowing, cycling, triathlon, and weightlifting. Experimental Aging Research, 36(4), 453-477. https://doi.org/10.1080/0361073X.2010.507433

Bertollo, M., Saltarelli, B. y Robazza, C. (2009). Mental preparation strategies of elite modern pentathletes. Psychology of Sport and Exercise, 10(2), 244-254. https://doi.org/10.1016/j.psychsport.2008.09.003

Buceta, J. M. (1990). Aspectos a tener en cuenta en relación con las deportistas españolas de alta competición. Seminario Mujer y Deporte, organizado por el Consejo Superior de Deportes, la Secretaría de Estado de Educación y el Instituto de la Mujer en Madrid. Publicado posteriormente en Planificación del Entrenamiento Deportivo y Rendimiento: Un enfoque interdisciplinar. Málaga: Instituto Andaluz del Deporte, 1995.

Buceta, J. M. (1994). El control del nivel de activación en los deportistas: experiencia en baloncesto de alto rendimiento. Apunts: Educación Física y Deportes, 35(1), 26-31

Buceta, J. M. (1996). Psicología y lesiones deportivas: prevención y recuperación. Dykinson. 
Buceta, J. M. (1998). Psicología del entrenamiento deportivo. Dykinson.

Campos, G. G., Valdivia-Moral, P., Zagalaz, J. C., Ortega, F. Z. y Romero, O. (2017). Influencia del control del estrés en el rendimiento deportivo: la autoconfianza, la ansiedad y la concentración en deportistas. RETOS. Nuevas Tendencias en Educación Física, Deporte y Recreación, 32(2), 3-6.

Cano, L. A., Alarcón, F., Ureña, N. y Piñar, M. (2009). Relación entre la ansiedad y la eficacia de un equipo de baloncesto durante la competición. Cuadernos de Psicología del Deporte, 9(Sup.), 51. https://revistas.um.es/cpd/article/view/85651

Carmona, A. (2019). Entrenamiento interválico de alta intensidad para la mejora en Remoergómetro [Tesis Doctoral no publicada]. Universidad de Sevilla, España.

Carrasco, A. E. R., Campbel, R. Z., García-Mas, A., Brustad, R. J., Quiroz, R. G. y López, A. L. (2010). Estrategias de afrontamiento y bienestar psicológico en jóvenes tenistas de competición. Revista de Psicología del Deporte, 19(1), 117-133.

Carretero, C. M., Moreno-Murcia, J. A., González, I., Ponce, J. J. y Gimeno, E. M. C. (2012). Ansiedad estado precompetitiva en judocas. Revista de Artes Marciales Asiáticas (RAMA), 7(1), 26-43.

Cox, R., Martens, M. y Russell, W. (2003). Measuring Anxiety in Athletics: The Revised Competitive State Anxiety Inventory-2. Journal of Sport and Exercise Psychology 25(4), 519-533. https:// doi.org/10.1123/jsep.25.4.519

Duval, F. (2010). Neurobiología del estrés. Revista Chilena Neuro-Psiquiátrica, 48(4), 307-318. https://doi.org/10.4067/S071792272010000500006

Elliott, B., Lyttle, A. y Birkett, O. (2002). The RowPerfect ergometer: A training aid for on-water single scull rowing. Sports Biomechanics, 1(2), 123-134. https://doi.org/10.1080/14763140208522791

Filaire, E., Sgnol, M., Ferrand, C., Maso, F. y Lac. G- (2001). Psychophysiological stress in judo during competitions. Journal of Sports Medicine and Physical Fitness, 47(2), 263-268.

Fonseca-Pedrero, E., Paino, M., Sierra-Baigie, S., Lemos-Giráldez, S. y Muñiz, J. (2012). Propiedades psicométricas del "Cuestionario Ansiedad Estado-Rasgo" (STAI) en universitarios. Behavioral Psychology, 20(3), 547-561.

García, E. M., Rodríguez, M., Andrade, E. M., y Arce, C. (2006). Adaptación del cuestionario MSCI para la medida de la cohesión en futbolistas jóvenes españoles. Psicothema, 18(3), 668-672.

García, R. H., Zafra, A. O. y Toro, E. O. (2008). Ansiedad y autoconfianza de jóvenes judokas en situaciones competitivas de alta presión. Análise Psicológica, 26(4), 689-696.

García-Fernández, J. M., Inglés, C. J., Díaz, Á., Lagos San Martín, N., Torregrosa, M. S. y Gonzálvez, C. (2016). Capacidad predictiva de la autoeficacia académica sobre las dimensiones del autoconcepto en una muestra de adolescentes chilenos. Estudios sobre Educación, 30(2), 31-50. https://doi. org/10.15581/004.30.31-50

García-Más, A., Palou, P., Smith, R. E., Ponseti, X., Almeida, P., Lameiras, J. y Leiva, A. (2011). Ansiedad competitiva y clima motivacional en jóvenes futbolistas de competición, en relación con las habilidades y el rendimiento percibido por sus entrenadores. Revista de Psicología del Deporte, 20(1), 197-207.

García-Naveira, A. (2017). Entrenamiento psicológico para la mejora del autocontrol en un entrenador de fútbol. Acción Psicológica, 14(1), 27-42. https://doi.org/10.5944/ap.14.1.19252
Galloway, M. T., Kadoko, R. y Jokl, P. (2002). Effect of aging on male and female master athletes' performance in strength versus endurance activities. American Journal of Orthopedics, $37(2), 93-98$.

Gentil, P., de Lira, C., Cardoso, S., Teixeira, C., Steele, J., Fisher, J. y Campos, M. (2017). El entrenamiento a intervalos de alta intensidad no afecta el aumento de fuerza en respuesta al entrenamiento de resistencia en mujeres premenopáusicas. Revista Europea de Fisiología Aplicada, 117(6), 1257-1265.

Gil, M., Capafons, B. y Labrador, E. (1993). Variables físicas y psicológicas predictoras del rendimiento deportivo y del cambio terapéutico. Psicothema, 5(1), 97-110.

Gimeno, F., Buceta, J. M. y Pérez-Llantada, M. C. (2001). El cuestionario Características Psicológicas Relacionadas con el Rendimiento Deportivo (CPRD): Características psicométricas. Análise Psicológica, 1(19), 93-113.

Gimeno, F., Buceta, J. M. y Pérez-Llantada, M. C. (2007). Influencia de las variables psicológicas en el deporte de competición: evaluación mediante el cuestionario Características psicológicas relacionadas con el rendimiento deportivo. Psicothema, 19(4), 667-672

González, P., y Andrés, H. (2019). Efectos de la aplicación de la Técnica de Relajación Muscular Progresiva de Jacobson sobre los niveles de estrés y alteraciones del sueño percibidas por los docentes de primaria y secundaria de la Unidad Educativa "Julio Verne" ([Tesis de Grado no publicada]. Universidad Central de Ecuador, Quito, Ecuador.

Gould, D., Eklund, R. C. y Jackson, S. A. (1993). Coping strategies used by U.S. Olympic wrestlers. Research Quarterly of Exercise and Sport, 64(1), 83-93.

Gutiérrez, M., Estévez, A., García, J. y Pérez, H. (1997). Ansiedad y rendimiento atlético en condiciones de estrés: efectos moduladores de la práctica. Revista de Psicología del Deporte, 6(2), 27-46.

Hagerman, F. C., Conors, M. C., Gault, J. A., Hagerman, G. R. y Polinski, W. J. (1978). Energy expenditure during simulated rowing. Journal of Applied physiology: respiratori, environmental and exercise physiology, 45(1), 87-93. https://doi.org/10.1152/ jappl.1978.45.1.87

Hardy, L., Jones, G. y Gould, D. (1996). Understanding psychological preparation for sport: Theory and practice of elite performers. Jones Wiley \& Sons.

Hatzigeorgiadis, A., Zourbanos N., Mpoumpaki, S. y Theodorakis, $Y$. (2009). Mechanisms underlying the self-talk-performance relationship: The effects of self-talk on self-confidence and anxiety. Psychology of Sport and Exercise, 10(1), 186-192. https://doi. org/10.1016/j.psychsport.2008.07.009

Huang, C. J., Nesser, T. W. y Edwards, J. E. (2007). Strength and power determinants of rowing performance. Journal of Exercise Physiology Online, 10(4), 43-50.

Jaenes. J. C. y Caracuel. J. C. (2005). Maratón. Preparación psicológica para el entrenamiento y la competición. Almuzara.

Jones, G. y Hardy, L. (1990). Stress in sport: Experiences of some elite performers. En G. Jones y L. Hardy (Eds.), Stress and performance in sport (pp. 247-277). John \& Sons.

Kendall, K. L., Smith, A. E., Fukuda, D. H., Dwyer, T. R. y Stout, J. R. (2011). Critical velocity: a predictor of 2000-m rowing ergometer performance in NCAA D1 female collegiate rowers. Journal 
of Sports Sciences, 29(9), 945-950. https://doi.org/10.1080/026 40414.2017.571274

Labrador, F. J., (2014). Entrenamiento en autoinstrucciones. En F. Labrador (Ed.), Técnicas de modificación de conducta (pp. 453458). Pirámide.

Latinjak, A. T., Álvarez, M. T. y Renom, J. (2009). Aplicando el auto-habla al tenis: su impacto sobre el foco atencional y el rendimiento. Cuadernos de Psicología del Deporte, 9(2), 19-19.

Lazarus, R. S. y Folkman, S. (1984). Estrés y procesos cognitivos. Martínez Roca.

Ley Orgánica 3/2018, de 5 de diciembre, de Protección de Datos Personales y garantía de los derechos digitales. Boletín Oficial del Estado (España), 294 de 6 de diciembre de 2018.

Loehr, J. E. (1984). How to overcome tension and play at your peak all the time. Tennis, 19(1), 66-76.

Mahoney, M. J., Gabriel, T. J., y Perkins, T. S. (1987). Psychological skills and exceptional athletic performance. The Sport Psychologist, 1(3), 181- 199.

Mamassis, G. y Dogamis, G. (2004). The effects of a mental training program on juniors pre-competitive anxiety, self-confidence, and tennis performance. Journal of Applied of Sport Psychology, 16(2), 118-137. https://doi. org/10.1080/10413200490437903

Marco, F.G., Buceta J. M y Pérez-Llantada. (2007). Influencia de las variables psicológicas en el deporte de competición: evaluación mediante el cuestionario Características psicológicas relacionadas con el rendimiento deportivo. Psicothema, 19(4), 667-672.

Márquez, S. (2004). Ansiedad, Estrés y Deporte. EOS.

Martens, R. (1987). Coaches Guide to Sport psychology. Human Kinetics.

Mosconi, S., Correche, M. S., Rivarola, M. F. y Penna, F. (2007). Aplicación de la técnica de relajación en deportistas para mejorar su rendimiento. Fundamentos en Humanidades, 16(2), 183-197.

Navarro-Guzmán, J. I., Amar, J. R. y González-Ferreras, C. (1995). Ansiedad pre-competitiva y conductas de autocontrol en jugadores de fútbol. Revista de Psicología del Deporte, 4(2), 7-17.

Olmedilla, A., Sánchez-Aldeguer, M., Alamansa, C., Gómez-Espejo, V. y Ortega, E. (2018). Entrenamiento psicológico y mejora de aspectos psicológicos relevantes para el rendimiento deportivo en jugadoras de fútbol. Revista de Psicología Aplicada al Deporte y al Ejercicio Físico, 3(1), Artículo e8. https://doi.org/70.5093/ rpadef2018a2

Ortín, F. J., De la Vega, R. y Gosálvez, J. (2013). Optimismo, ansiedad estado y autoconfianza en jugadores de balonmano. Anales de Psicología, 29(3), 637-641. https://doi.org/10.6018/ analesps.29.3.175751

Perna, S., Bologna, Ch., Agosti, I. y Rondanelli (2017). High intensity crossfit training compared to high intensity swimming: a pre-post trial to assess the impact on body composition, muscle strength and resting energy expenditure. Asian Journal of Sports Medicine, 9(1). https://doi.org/10.5812/asjsm. 13843

Pierna, J., Abad, F., Carabaza, J. y Sancho, N. (2008). El entrenamiento en banco fijo: utilidad del remoergómetro. Deporte y Actividad Física para Todos, 4(1), 121-130.

Portilla, F. J. (2016). Ansiedad competitiva y técnicas de afrontamiento en 27 futbolistas profesionales de la Universidad Internacional del Ecuador [Tesis Doctoral no publicada]. Universidad Internacional, Ecuador.
Pozo, A. (2007). Intensidad y dirección de la ansiedad competitiva y expectativas de resultado en atletas y nadadores. Revista de Psicología del Deporte, 16(2), 137-150.

Ries, F., Castañeda, C., Campos, M. C. y Del Castillo, O. (2012). Relación entre ansiedad-rasgo y ansiedad-estado en competiciones deportivas. Cuadernos de Psicología del Deporte, 12(2), 9-16.

Roberts, G.C. (Ed.). (2001). Advances in motivation in sport and exercise. Human Kinetics.

Ruiz-Juan, F., Sancho, A. Z. y Flores-Allende, G. (2015). Variables predictoras de la ansiedad precompetitiva: aspectos diferenciales en corredores de fondo en ruta. Universitas Psychologica, 14(3), 1021-1031. https://doi.org/10.11144/Javeriana.upsy74-3.vpap

Salas, J. D. Z. y Salazar, W. (2004). Efecto de la tensión, ansiedad y relajación con respecto al rendimiento cognitivo en deportistas. Cuadernos de Psicología del Deporte, 4(2), 91-100.

Sánchez-Hernández, M. y López-Fernández, M. (2005). Pigmalión en la escuela. Editorial Universidad Autonómica de la Ciudad de México.

Sánchez, J. C. J., Gómez, R. P., Dueñas, K. G. N. y Gómez-Millán, M. R. B. (2012). Ansiedad y autoconfianza precompetitiva en triatletas. Revista Iberoamericana de Psicología del Ejercicio y el Deporte, 7(1), 113-124.

Saz, A. I. (2000). Diccionario de Psicología. Madrid: Libro Hobby-Club. Telletxea, S. (2007). Aplicación de un programa de intervención psicosocial orientado hacia la formación y optimización de las capacidades de rendimiento en deportistas [Tesis Doctoral no publicada]. Universidad del País Vasco, España.

Scanlan, T. K., Babkes, M. L. y Scanlan, L. A. (2005). Participation in sport: A developmental glimpse at emotion. En J. L. Mahoney, R. W. Larson y J. S. Eccles (Eds.), Organized activities as contexts of development: Extracurricular activities, after school, and community programs (pp. 275-309). Erlbaum.

Schabort, E.J., Hawley, J.A., Hopkins, W. G. y Blum, H. (1999). High reliability of per-formance of well-trained rowers on a rowing ergometer. Journal of Sports Sciences, 17, 627-632. https://doi. org/10.1080/026404199365650

Schlatter, J. (2003). La ansiedad. Un enemigo sin rostro. Eunsa Editorial.

Shavelson, J., Hubner, J. J. y Stanton C. G. (1976). Review of Educational Research, 46(3), 407-442.

Sierra, J. C., Ortega, V. y Zubeidat, I. (2003). Ansiedad, angustia y estrés: tres conceptos a diferenciar. Revista Mal-estar e Subjetividade, 3(1), 10-59.

Silva, T. (2019). Construcción de cartas centiles y valores normativos para el rendimiento en remo indoor [Tesis Doctoral no publicada]. Universidad de Vigo, España.

Smith, R. (1989). Athletic stress and burnout: Conceptual models and intervention strategies. En D. Hackfort y C. D. Spielberger (Eds.), Anxiety in sports: An international perspective (pp. 183201). Hemisphere

Smith, J. (1992). Entrenamiento cognitivo conductual para la relajación. Desclée de Brouwer, S.A.

Spielberger, C. D. (1966). Anxiety and Behavior. Academic.

Torrez, M. y Núñez, L. (2012). Fútbol, ansiedad y psicología deportiva [Tesis Doctoral no publicada]. Universidad del Valle, Colombia.

Urra, B. (2014). Evaluación de la efectividad del entrenamiento de estrategias de afrontamiento en el nivel de ansiedad precompetitiva en tenistas. Revista de Psicología del Deporte, 23(1), 67-74. 
Vargas, J. G. (2015). El efecto Pigmalión y su efecto transformador a través de las expectativas. Perspectivas Docentes, 57(1), 40-43.

Wang, J., Marchant, D., Morris, T. y Gibbs, P. (2004). Self-consciousness and trait anxiety as predictors of choking in sport. Journal Science Medicine Sport, 7(2), 174-185. https://doi.org/10.1016/ s1440-2440(04)80007-0

Weinberg, G. (2007). Fundamentos de psicología del deporte y del ejercicio físico. En D. G. Robert Weinberg, Fundamentos de Psicología del Deporte y del Ejercicio Físico (pp, 286-287). Medica Panamericana.
Zainuddin, F., Umar, M., Razman, R. y Shaharudin, Sh. (2019). Changes of lower limb kinematics during 2000m. ergometer rowing among male Junior National Rowers. Pertanika Journal of Social Science and Humanities, 27(3), 2169-2184

Zinsser, N., Bunker, L. y Williams, J. M. (2006). Cognitive techniques for building confidence and enhancing performance. En J. M. Williams (Ed.), Applied sport psychology: Personal growth to peak performance (5th Ed., pp. 349-381). McGraw-Hill, Inc, Higher Education. 